\title{
Ringer's lactate is compatible with the rapid infusion of AS-3 preserved packed red blood cells
}

\section{Le lactate Ringer est compatible avec une perfusion rapide de culots globulaires préservés avec l'AS-3}

\author{
Khala Albert, BSc $\cdot$ Janet van Vlymen, MD • \\ Paula James, MD $\cdot$ Joel Parlow, MD \\ Received: 3 September 2008/Revised: 19 December 2008/Accepted: 12 January 2009/Published online: 2 April 2009 \\ (C) Canadian Anesthesiologists' Society 2009
}

\begin{abstract}
Purpose Ringer's lactate (RL) may be preferable to normal saline (NS) for large volume resuscitation. Authorities recommend against its mixture with packed red blood cells (PRBC) due to a theoretical risk of clotting. The purpose of this study was to test whether RL, as compared with NS, leads to a risk of clotting when used to dilute AS-3 preserved PRBC in a clinically relevant model.

Methods Following Ethics Board approval, eight units of unused, unexpired AS-3 preserved PRBC were obtained. Four sets of two parallel studies were performed, comparing RL with NS as the diluent for PRBC. The mixtures were infused using gravity flow through standard blood filter tubing and fluid warmer, to simulate an intraoperative blood transfusion. A series of progressively more dilute samples was collected. These were filtered and analyzed macroscopically, then using enzyme-linked immunosorbent assay to quantify the amount of F1+2 (the breakdown products of thrombin generation).
\end{abstract}

An abstract of this research was presented at the Annual Meeting of the Canadian Anesthesiologists' Society in June, 2008. It was awarded the Anemia Institute for Research and Education Travel Award.

This article is accompanied by an editorial. Please see Can J Anesth 2009; 56: 5.

K. Albert, BSc · J. van Vlymen, MD · J. Parlow, MD ( $)$ Department of Anesthesiology, Queen's University and Kingston General Hospital, 76 Stuart Street, Kingston, ON K7L 2V7, Canada

e-mail: parlowj@queensu.ca

P. James, MD

Division of Hematology, Queen's University and Kingston General Hospital, Kingston, ON, Canada
Results No filters in any of the NS and RL mixtures contained evidence of clot or debris. In the NS group, the F1+2 levels ranged from 2.7 to $38.0 \mathrm{pmol} \cdot L^{-1}$. In the RL group, the $F 1+2$ levels ranged from 3.2 to $289.7 \mathrm{pmol} \cdot L^{-1}$. All of these values were below the physiologic levels determined in previous studies.

Conclusion In this simulation of intraoperative blood transfusion, RL did not lead to visible or molecular evidence of activation of the clotting cascade. The current study challenges recommendations that AS-3 PRBC should not be mixed with $R L$ for rapid transfusion.

\section{Résumé}

Objectif L'utilisation de lactate Ringer (LR) pourrait être préférable à une solution salée isotonique (SSI) de chlorure de sodium pour la réplétion liquidienne majeure. Les autorités déconseillent le melange de LR avec des culots globulaires en raison d'un risque théorique de coagulation. L'objectif de cette étude était de tester si le LR, comparativement au SSI, provoque un risque de coagulation lorsqu'il est utilisé pour diluer un culot globulaire préservé avec l'AS-3 dans un modèle cliniquement pertinent.

Méthode Après avoir reçu le consentement du Comité d'éthique, huit unités de culots globulaires avec préservatif AS-3 non utilisés et non périmés ont été obtenues. Quatre ensembles de deux études parallèles ont été réalisés comparant le LR avec le SSI comme diluant des culots globulaires. Les melanges ont été perfusés par écoulement par gravité via une tubulure de filtration standard et un chauffe-soluté afin de simuler une transfusion sanguine peropératoire. Une série d'échantillons de plus en plus dilués a été récoltée. Ces échantillons ont été filtrés et analysés par macroscopie, puis à l'aide d'un dosage immunoenzymatique sur support solide (ELISA) afin de quantifier la quantité de fragments de la prothrombine 
$F 1+2$ (les produits de dégradation de la génération de thrombine).

Résultats Aucun des filtres utilisés dans les mélanges SSI ou LR ne contenait de traces de coagulation ou de débris. Dans le groupe SSI, les niveaux de FI+2 s'échelonnaient de 2,7 à $38,0 \mathrm{pmol} \cdot \mathrm{L}^{-1}$. Dans le groupe $L R$, les niveaux de $F 1+2$ s'échelonnaient entre 3,2 et $289,7 \mathrm{pmol} \cdot \mathrm{L}^{-1}$. Ces valeurs se situaient toutes au-dessous des niveaux physiologiques définis dans les études précédentes.

Conclusion Dans le cadre de cette simulation d'une transfusion sanguine peropératoire, le LR n'a pas engendré de traces visibles ou moléculaires d'activation de la cascade de coagulation. Cette étude remet en question les recommandations selon lesquelles les PRBC AS-3 ne devraient pas être mélangés au LR pour la transfusion rapide.

Left untreated, continued rapid loss of blood volume is associated with $100 \%$ mortality. ${ }^{1}$ The crystalloid solutions, normal saline (NS) and Ringer's lactate (RL), are commonly used as first-line volume replacement solutions in severe blood loss. ${ }^{1}$ Normal saline contains non-physiologic sodium and chloride concentrations of $154 \mathrm{mEq} \cdot \mathrm{L}^{-1}$, while $\mathrm{RL}$ contains physiologic concentrations of sodium, chloride, potassium, calcium, and lactate. Although inexpensive and accessible, administering large volumes of NS, but not RL, has been shown to cause hyperchloremic metabolic acidosis and disturbances in electrolyte homeostasis. ${ }^{2-5}$ Thus, it has been suggested that RL is the initial fluid of choice for resuscitating the patient in hemorrhagic shock. ${ }^{2,3,6}$

Crystalloids are generally co-administered with packed red blood cells (PRBC) in order to prime and flush intravenous tubing and often to dilute the blood to improve flow characteristics. ${ }^{7,8}$ Current standards set by Canadian Blood Services (CBS) and the American Association of Blood Banks (AABB) state that only NS should be used to dilute PRBC. ${ }^{9,10}$ These guidelines are based, in part, on research completed in the 1970s and 80s which showed that using calcium-containing crystalloids, such as RL, can cause clotting when combined with PRBC preserved with citratephosphate-dextrose-adenine (CPDA). ${ }^{11,12}$ However, a number of studies have challenged the claim that it is unsafe to use RL to reconstitute PRBC, and to date, no study has shown RL to cause clotting in clinically relevant dilutions of PRBC to crystalloid. ${ }^{2,6,8,13}$ It has been shown that mixtures of PRBC and crystalloid, in a ratio of $2: 1$ or higher, do not overwhelm the calcium-chelating ability of the citrate in the CPDA preservative solution. ${ }^{14}$

Although the concentration of crystalloid used to dilute PRBC would be limited by the capacity of the blood bags, PRBC are exposed to higher dilutions of RL when intravenous tubing is flushed following transfusion.
A newer additive solution, AS-3, is commonly used to preserve packed red cells following anticoagulation with CP2D. This solution contains less plasma and more citrate than CPDA, and also consists of dextrose, adenine, sodium citrate, sodium phosphate, citric acid, and sodium chloride in water. There are no published reports to date examining the safety of mixing RL with AS-3 PRBC. The purpose of the current study was to test whether RL causes evidence of clotting when combined with AS-3 PRBC in a clinically relevant model of blood dilution and transfusion.

\section{Methods}

Following approval by the Queen's University Research Ethics Board, the Blood Transfusion Service at Kingston General Hospital supplied eight units of PRBC that were not suitable for clinical use. These blood units were prepared by the Canadian Blood Service by adding the anticoagulant, $\mathrm{CP} 2 \mathrm{D}$, to whole blood, centrifuging to obtain PRBC, then adding $100 \mathrm{~mL}$ of AS-3 for preservation. All of the units of PRBC were within the 42-day viability period, and all information identifying the patients was removed prior to distribution. The hematocrit of the units ranged from 0.55 to 0.65 , with a volume ranging from 300 to $350 \mathrm{~mL}$. In a similar manner to the clinical environment, the units of PRBC were maintained at $4{ }^{\circ} \mathrm{C}$ and the crystalloids were maintained at room temperature prior to experimentation. The intravenous tubing was initially primed with crystalloid, and the bags of PRBC were diluted with $150 \mathrm{~mL}$ of crystalloid (Fig. 1), providing a hematocrit ranging from 0.41 to 0.49 prior to infusion. Immediately after mixing, the solutions of PRBC and crystalloid were infused by gravity through standard blood filter tubing with a 170 micron filter (Baxter Corporation, Toronto, ON, Canada) from an elevation of $1.5 \mathrm{~m}$. A measuring chamber (Buratrol $^{\circledR}$, Travenol, Toronto, ON, Canada) facilitated accurate measurements of volumes. The solutions were warmed with a $2.4 \mathrm{~m}$ fluid warming line at $37^{\circ} \mathrm{C}$ (Hotline ${ }^{\circledR}$ Blood and Fluid Warmer, Smiths Medical, Rockland, MA, USA). A stopcock was attached to the end of the warming line to facilitate collection of samples. Based on a model of rapid blood transfusion, all trials were completed in less than $30 \mathrm{~min}$. A total of eight experiments (four trials, each using RL and NS in parallel as diluents) were performed All filters, intravenous tubing, and collection containers were changed between experiments.

As each solution was infused, seven $20 \mathrm{~mL}$ samples of increasingly dilute blood were drawn from the end of the warming tubing. Sample 1 consisted of the original solution of PRBC diluted with $150 \mathrm{~mL}$ of crystalloid. The tubing was then flushed with $75 \mathrm{~mL}$ of crystalloid to obtain Sample 2. Samples 3, 4, 5, 6, and 7 were each obtained 
Fig. 1 Graphic representation of blood transfusion model

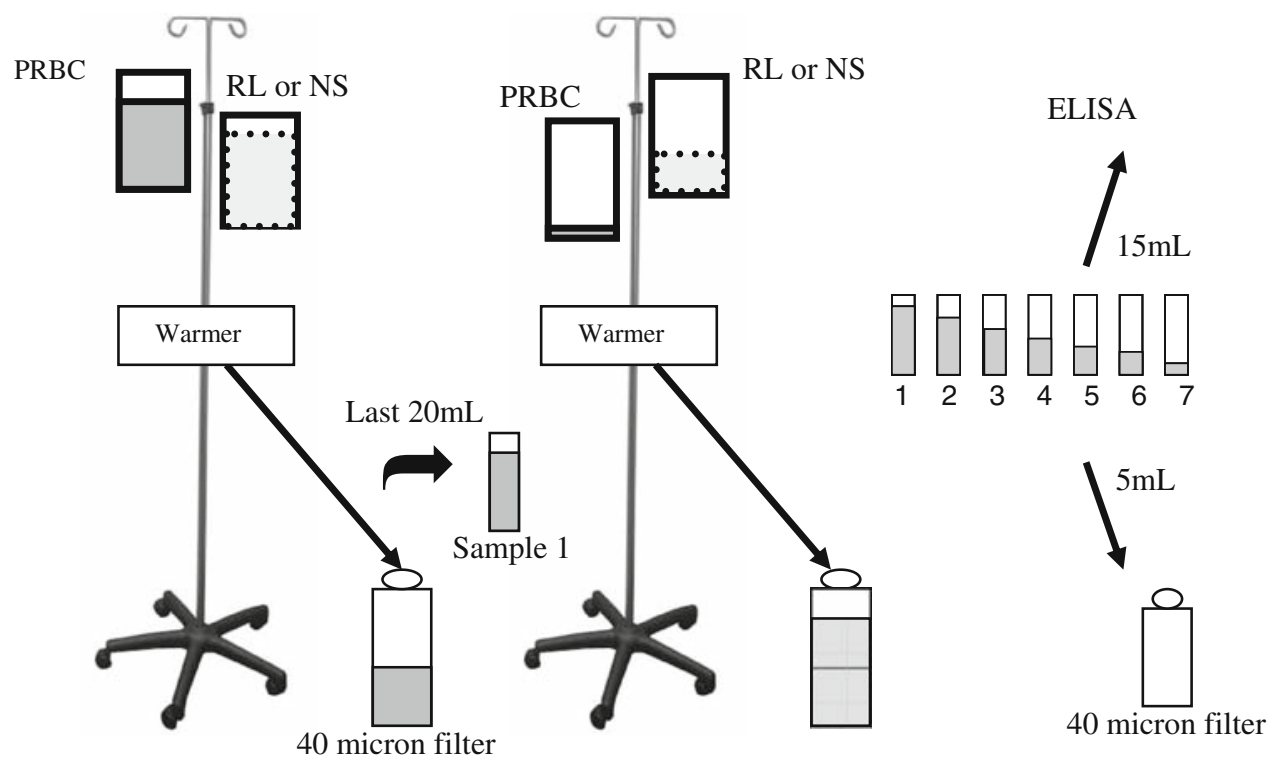

following flushing with a further $25 \mathrm{~mL}$ of crystalloid. Thus, the seven samples were progressively diluted with crystalloid to yield dilutions of crystalloid:PRBC ranging from approximately $1: 2$ to $10: 1$. Each of these $20 \mathrm{~mL}$ samples was then divided for analysis using both macroscopic and molecular techniques.

For macroscopic analysis, the serially diluted samples were passed through 40 micron filters and visually examined for the presence of clots or debris. For molecular analysis, the samples were centrifuged at $4^{\circ} \mathrm{C}$ for $30 \mathrm{~min}$ at a speed of $4750 \mathrm{rpm}$ to obtain platelet poor plasma. Thrombin generation was measured using an enzyme-linked immunosorbent assay (ELISA) technique that quantifies the amount of $F 1+2$, the breakdown products of thrombin generation $\left(\right.$ Enzygost $^{\circledR} \mathrm{F} 1+2$, Behring Diagnostics Inc., Westwood, MA, USA). This assay is used clinically to assess whether ongoing clot formation is occurring in vivo or in vitro. Physiologic levels of $\mathrm{F} 1+2$ indicate no activation of the coagulation pathway. Conversely, the detection of high levels of $\mathrm{F} 1+2$ allow quantification of the magnitude of clotting that has occurred. For each experiment, the seven samples of progressively more dilute PRBC were run in duplicate. In order to quantify the samples, a 4-point standard curve was generated using duplicate standard concentrations of $5,10,20$, and $40 \mathrm{pmol} \cdot \mathrm{L}^{-1}\left(R^{2}=0.949\right)$. In addition, controls of 5,10 , and $50 \mathrm{pmol} \cdot \mathrm{L}^{-1}$ were used to assure the validity of the results.

\section{Results}

The eight units of PRBC used in the experiments ranged in age from 6 to 35 days old. Upon visual inspection of the 40 micron filters, none of the dilutions led to any macroscopic evidence of clot or debris in either the NS or the RL samples. All filters continued to allow free passage of blood-crystalloid mixtures.

Using the ELISA technique, none of the samples of either NS or RL showed evidence of thrombin activation (Fig. 2). In the NS group, the F1+2 levels ranged from 2.7 to $38.0 \mathrm{pmol} \cdot \mathrm{L}^{-1}$ (Fig. 2). In the RL group, the $\mathrm{F} 1+2$
Fig. 2 Mean $\mathrm{F} 1+2$ in duplicates of increasingly dilute mixtures of AS-3 packed red blood cells and crystalloid (NS or RL). Area enclosed by dashed lines corresponds to physiologic $\mathrm{F} 1+2$ levels in healthy volunteers ${ }^{15}$

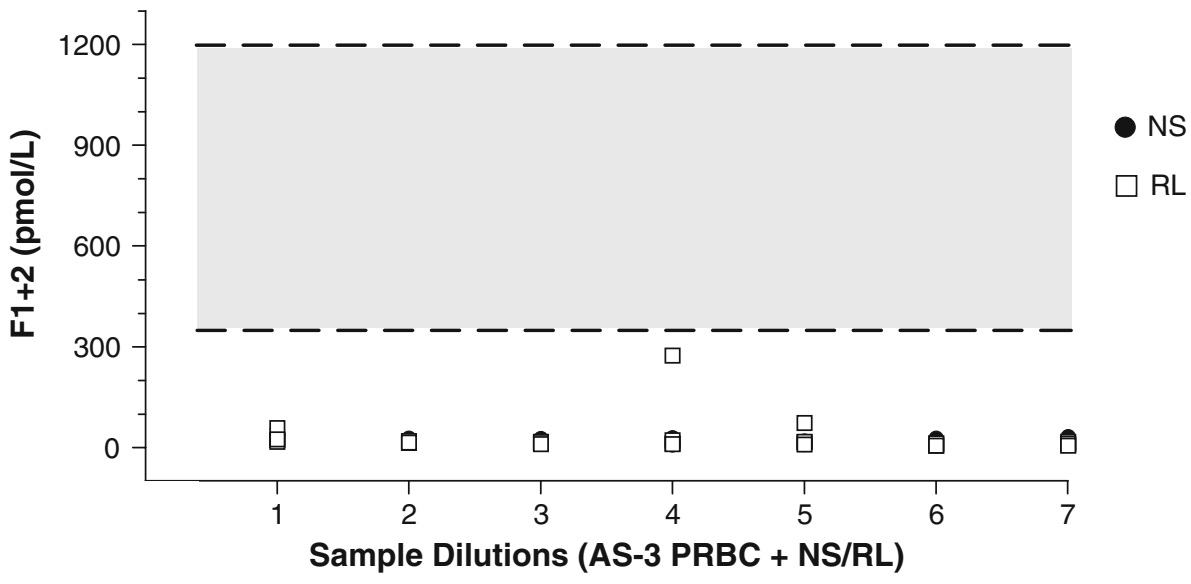


levels ranged from 3.2 to $289.7 \mathrm{pmol} \cdot \mathrm{L}^{-1}$. All values were below normal physiologic levels, as determined by Pelzer et al ${ }^{15}$ Control measurements confirmed the validity of the technique.

\section{Discussion}

The additive solution, AS-3, is a frequently used preservative for PRBC. This medium is further protective of clot formation in that it contains more citrate and less serum than the previously used CPDA. There are no previously published reports examining the potential clotting tendency when using RL to dilute PRBC preserved with AS-3. The current study is novel in its approach to determine the compatibility of RL, in combination with PRBC preserved with AS-3, in a simulated clinical environment. Thus, the methodology was constructed to represent a "real-life" model of rapid blood transfusion.

Neither macroscopic nor molecular analysis revealed evidence of clotting with either NS or RL. At high dilutions, the detection of low levels of F1+2 showed that the amount of calcium contained in the RL up to dilutions of RL:PRBC of approximately 10:1 was insufficient to overwhelm the citrate present in the AS-3 preservative solution. The normal physiologic range of $\mathrm{F} 1+2$ was previously determined by Pelzer et al. ${ }^{15}$ immediately after withdrawal of blood in 95 healthy volunteers. None of the dilutions of blood with either RL or NS in our study exceeded the reference range of 320 to $1200 \mathrm{pmol} \cdot \mathrm{L}^{-1}$, implying that no clinically significant activation of the coagulation cascade occurred.

Metabolic acidosis is a frequent consequence of massive blood loss due to hypoperfusion and the increase in anaerobic metabolism. Resuscitation fluids can lower the acid load in two ways. Indirectly, the production of acid can be minimized by increasing the intravascular volume and maintaining adequate tissue perfusion. ${ }^{1}$ Directly, the acid load can be reduced if the fluid solution contains an acid buffer or a source of buffer. ${ }^{1}$ Where whole blood, plasma, or albumin solutions contain protein, which can act as a buffer, RL contains a potential source of bicarbonate. ${ }^{3}$

Normal saline is a hyperchloremic solution containing $154 \mathrm{mEq} \cdot \mathrm{L}^{-1}$ of chloride $\left(\mathrm{Cl}^{-}\right)$. Large volume saline infusion produces acidosis by directly diluting the bicarbonate $\left(\mathrm{HCO}_{3}{ }^{-}\right)$concentration and by increasing plasma chloride relative to plasma sodium. ${ }^{4}$ In the kidneys, a higher concentration of $\mathrm{Cl}^{-}$reciprocally leads to an increase in excretion of $\mathrm{HCO}_{3}{ }^{-}$contributing to the development of an anion gap metabolic acidosis. ${ }^{3}$ Studies performed in animals have shown that treatment of hemorrhagic shock with NS, when compared to balanced salt solutions or RL, leads to an exacerbation of pre-existing acidosis and increased mortality. ${ }^{4,16,17}$ This finding is significant in that acidosis is associated with impaired cardiac performance, decreased renal perfusion, and poor prognosis. ${ }^{2,3}$ It has also been shown to increase perioperative blood loss and to raise the level of circulating inflammatory cytokines, such as tumour necrosis factor and interleukins 6 and $10 .^{4,5}$

Thus, RL may be a more logical choice for resuscitation of hemorrhagic shock. ${ }^{2,3,6}$ It is postulated that RL may improve metabolic acidosis through in vivo conversion of its lactate component to circulating bicarbonate. ${ }^{6}$ The metabolism of lactate, however, does take time; thus, theoretically, it does not have an effect in acute hemorrhage. ${ }^{1,3}$ Some studies suggest that rapid and sufficient treatment of hypovolemic shock returns the $\mathrm{pH}$ to normal regardless of the crystalloid being administered. ${ }^{18}$ Despite this, there is considerable evidence that the use of RL to treat massive blood loss leads to significantly less morbidity and mortality than the use of NS. ${ }^{2,5,6,16,19}$

The CBS and AABB have long advised that only NS should be used to dilute PRBC. ${ }^{9,10}$ These standards are based on laboratory research conducted more than 20 years ago that have demonstrated that calcium-containing crystalloids could cause clotting to occur when combined with warmed blood components preserved with CPDA. ${ }^{11,12}$ These guidelines have remained despite numerous reports that have shown that RL does not cause clotting in clinically relevant dilutions. ${ }^{2,6,8,13}$ Blood preservatives utilize citrate to chelate calcium contained in blood products, thereby preventing activation of the clotting cascade. RL contains $0.020 \mathrm{~g} \cdot \mathrm{L}^{-1}$ of $\mathrm{CaCl}_{2}$, while one unit of CPDA-PRBC contains approximately $0.520 \mathrm{~g}$ of citrate. $^{2}$ When PRBC are diluted in a clinically relevant ratio of $2: 1$ or greater, the chelating capacity of the CPDA solution would not be exceeded.

A variety of methodologies have been used in previous studies to determine the presence or absence of clots, including weighing of filters, transfusion times, and the microscopic visualization of fibrin strands. Lorenzo et al. ${ }^{2}$ compared RL and NS with rapid infusion rates. They found that there was no significant difference in clot formation, filter weight, or infusion time between NS and RL. Following infusion through a filter at $540 \mathrm{~mL} \cdot \mathrm{h}^{-1}$, King et al. ${ }^{8}$ demonstrated no difference in filter weights between PRBC diluted with either RL or NS. This group further concluded that $0.23 \mathrm{mmol} \cdot \mathrm{L}^{-1}$ of ionized $\mathrm{Ca}^{2+}$ appeared to be the threshold for clot formation, and that this level could not be exceeded when PRBC units were diluted with RL in a ratio greater than $2: 1{ }^{8}$ Cull et al. ${ }^{13}$ showed no significant clot formation with the dilution of PRBC and RL at clinically relevant levels (5:1, 3:1, and 2:1). In previous experimentation, Parlow et al. ${ }^{14}$ visually examined filters to determine the presence or absence of clot formation. They reported the obvious presence of macroscopic clot debris in CPDA-PRBC samples diluted 
with more than $70 \%$ RL by volume and incubated for 30 $60 \mathrm{~min}$. No clot formation was visualized at clinically relevant ratios.

Rosenblatt et al..$^{20}$ diluted supernatant plasma from red cell concentrates with varying amounts of RL and used an ELISA technique to measure thrombin generation through quantification of the presence of $\mathrm{F} 1+2$. The presence of $\mathrm{F} 1+2$ was only detectable in very small quantities in the samples where the AS-3 PRBC supernatant was diluted with RL in a ratio of $1: 20$ and $1: 10$. In clinically relevant dilutions of $2: 1$ or higher, no F1+2 could be detected.

Limitations of the current study include lack of blinding in the analysis of clot formation. Although this part of the analysis was macroscopic and non-quantitative, previous studies had shown that any clotting caught by the filters would be readily obvious on visual inspection. The quantification of $\mathrm{F} 1+2$ by ELISA was conducted as a more objective and sensitive analysis of coagulation. Neither technique showed evidence of activation of the clotting cascade. Four repetitions of the parallel experiments comparing RL and NS were carried out. Although only eight units of blood were used in the analyses, the PRBC were diluted to an extent that the concentration of citrated blood was minimal in the terminal samples. Given the relative similarity in citrate concentration in stored blood, it would thus be unlikely that analyzing a larger sample of blood units would yield a different result. The current model was designed to simulate rapid blood transfusion as it is often carried out in the intraoperative setting. Crystalloid is not always necessary to dilute PRBC but is used in priming and flushing the blood tubing. In clinical settings where blood is transfused slowly, we recommend that NS be the crystalloid used for priming and flushing lines. Finally, the analyses done in the current study pertain to AS-3 preserved blood. Experiments would have to be repeated in blood stored with newer preservatives, such as CPD-SAGM (Canadian Blood Service), although the content of sodium citrate in these solutions is identical to that used in CP2D-AS-3 blood.

In conclusion, the current study supports that RL can be used to dilute AS-3 preserved PRBC for rapid transfusion without activation of the clotting cascade. Due to the potential benefits of utilizing RL for the resuscitation of hypovolemia, we suggest that a re-evaluation of existing guidelines be considered in regards to the use of RL to reconstitute AS-3 preserved PRBC.

Acknowledgments The authors sincerely thank Dr. Lois Shepherd, Yvette Chirinian, Carol Hegadorn, and the staff of the Blood Transfusion Service at Kingston General Hospital for their tremendous help with this study. This study was funded using departmental research support.

Conflicts of interest None declared.

\section{References}

1. Traverso $L W$, Medina $F$, Bolin RB. The buffering capacity of crystalloid and colloid resuscitation solutions. Resuscitation 1985; 12: 265-70.

2. Lorenzo M, Davis JW, Negin S, et al. Can Ringer's lactate be used safely with blood transfusions? Am J Surg 1998; 175: 30810.

3. Ho AM, Karmakar MK, Contardi LH, Ng SS, Hewson JR. Excessive use of normal saline in managing traumatized patients in shock: a preventable contributor to acidosis. J Trauma 2001; 51: 173-7.

4. Kellum JA, Song M, Almasri E. Hyperchloremic acidosis increases circulating inflammatory molecules in experimental sepsis. Chest 2006; 130: 962-7.

5. Boldt J, Haisch G, Suttner S, Kumle B, Schellhase F. Are lactated Ringer's solution and normal saline solution equal with regard to coagulation? Anesth Analg 2002; 94: 378-84.

6. Orlinsky M, Shoemaker W, Reis ED, Kerstein MD. Current controversies in shock and resuscitation. Surg Clin North Am 2001; 81: 1217-62, xi-xii.

7. Crosby ET. Perioperative haemotherapy: II. Risks and complications of blood transfusion. Can J Anaesth 1992; 39: 822-37.

8. King WH, Patten ED, Bee DE. An in vitro evaluation of ionized calcium levels and clotting in red blood cells diluted with lactated Ringer's solution. Anesthesiology 1988; 68: 115-21.

9. Brecher ME. Technical Manual. 14th ed. Bethesda, MD: American Association of Blood Banks Press; 2002.

10. Callum JL, Pinkerton PH. Blood Easy 2: Blood Transfusions, Blood Alternatives and Transfusion Reactions. 2nd ed. Sunnybrook \& Women's College Health Science Centre, 2005.

11. Edwards MP, Clark DJ, Mark JS, Wyld PJ. Compound sodium lactate (Hartmann's) solution. Caution: risk of clotting. Anaesthesia 1986; 41: 1053-4.

12. Ryden SE, Oberman HA. Compatibility of common intravenous solutions with CPD blood. Transfusion 1975; 15: 250-5.

13. Cull DL, Lally KP, Murphy KD. Compatibility of packed erythrocytes and Ringer's lactate solution. Surg Gynecol Obstet 1991; 173: 9-12.

14. Parlow JL, Johnson GD, Adams MA, Lillicrap DP. Compatibility of lactated Ringer's solution with packed red blood cells. Can J Anaesth 1989; 36: S77-8.

15. Pelzer H, Schwarz A, Stuber W. Determination of human prothrombin activation fragment $1+2$ in plasma with an antibody against a synthetic peptide. Thromb Haemost 1991; 65: 153-9.

16. Healey MA, Davis RE, Liu FC, Loomis WH, Hoyt DB. Lactated Ringer's is superior to normal saline in a model of massive hemorrhage and resuscitation. J Trauma 1998; 45: 894-9.

17. Traverso LW, Bellamy RF, Hollenbach SJ, Witcher LD. Hypertonic sodium chloride solutions: effect on hemodynamics and survival after hemorrhage in swine. J Trauma 1987; 27: 32-9.

18. Cervera AL, Moss G. Dilutional re-expansion with crystalloid after massive hemorrhage: saline versus balanced electrolyte solution for maintenance of normal blood volume and arterial $\mathrm{pH}$. J Trauma 1975; 15: 498-503.

19. Coran AG, Ballantine TV, Horwitz DL, Herman CM. The effect of crystalloid resuscitation in hemorrhagic shock on acid-base balance: a comparison between normal saline and Ringer's Lactate solutions. Surgery 1971; 69: 874-80.

20. Rosenblatt M, Heddle NM, Kelton JG, Klama L, Hayward C. Evaluation of clot formation in AS-3 red cells diluted with Ringer's lactate. Transfusion 2000; 40: 132S. 